\title{
Biomarkers for gastric cancer: Progression in early diagnosis and prognosis (Review)
}

\author{
ZILIANG JIN, WEIHUA JIANG and LIWEI WANG \\ Department of Oncology, Shanghai Key Laboratory of Pancreatic Diseases, \\ Shanghai Jiaotong University Affiliated First People's Hospital, Shanghai 200080, P.R. China
}

Received April 3, 2014; Accepted January 8, 2015

DOI: $10.3892 / \mathrm{ol} .2015 .2959$

\begin{abstract}
Gastric cancer is one of leading causes of cancer-related mortality worldwide and is a notable disease due to its heterogeneity. Recently, numerous studies have investigated the molecular basis of gastric cancer, involving the alteration of pathogenesis, and invasion and metastasis. With the development of modern technologies, various novel biomarkers had been identified that appear to possess diagnostic and prognostic value; therefore, the present review describes our current knowledge of biomarkers for the early diagnosis and prognosis of gastric cancer. Classic biomarkers for gastric cancer diagnosis include carcinoembryonic antigen and cancer antigen 19-9, while microRNA and DNA hypomethylation are proposed as novel biomarkers. Excluding classical biomarkers, biomarkers for determining the progression and prognosis of gastric cancer focus on targeting microRNAs, epigenetic alterations and genetic polymorphisms.
\end{abstract}

\section{Contents}

1. Introduction

2. Biomarkers for early diagnosis

3. Prognostic biomarkers

4. Conclusion

\section{Introduction}

A total of 989,600 new cases of gastric cancer and 738,000 gastic cancer related mortlities are estimated to have occurred in 2008 worldwide, accounting for $8 \%$ of the total cases and $10 \%$ of

Correspondence to: Dr Liwei Wang, Department of Oncology, Shanghai Key Laboratory of Pancreatic Diseases, Shanghai Jiaotong University Affiliated First People's Hospital, 100 Haining Road, Shanghai 200080, P.R. China

E-mail: jz1920222@126.com

Key words: gastric cancer, early diagnosis, prognosis, biological marker total mortalies due to cancer worldwide (1). The incidence and mortality rates of gastric cancer have decreased overall in recent years, however, gastric cancer remains the leading cause of cancer-related mortality in developing countries (1). Despite progression in the diagnosis and treatment of advanced gastric cancer, the prognosis of gastric cancer patients remains poor, in part due to the low rate of diagnosis during its early stages. Numerous studies investigating the molecular mechanisms of gastric cancer invasion and metastasis have identified that survival is associated with the ability of the cancer to metastasize (2-4). Standard biomarkers used for gastric cancer diagnosis include carcinoembryonic antigen (CEA) and cancer antigen 19-9 (CA19-9) (5), however, recently microRNA and DNA hypomethylation have been proposed as novel biomarkers $(6,7)$. TNM staging (tumor, lymph nodes and metastasis) was commonly used to assess patient prognosis in developed and developing countries. However, the approach was insufficient, because prognosis often varies between patients at the same tumor stage (8). The current review focuses on the currently available biomarkers for the early diagnosis and prognosis of gastric cancer.

\section{Biomarkers for early diagnosis}

Classical biomarkers

$C E A$. CEA was initially identified in 1965 (9) and was first applied for the diagnosis of early gastric cancer in 1980 (10). CEA is currently regarded as the most valuable serum protein marker for identifying patients at risk of developing gastric cancer and for the diagnosis of early stage gastric cancer. However, serum CEA can be detected in patients with alternative types of carcinoma, thus, it exhibits low specificity and sensitivity.

CA 19-9. CA 19-9 has previously been a commonly used marker in gastrointestinal cancer; however, it is present in a number of types of cancer, in particular pancreatic and gastric cancer. The CA 19-9 test in combination with the CEA test is a useful adjunct for monitoring carcinoma of the stomach; however, the sensitivity of performing these assays concurrently is comparable to performing the CEA assay alone in gastric carcinoma (11).

\section{Novel biomarkers}

microRNA (miR/miRNA). In gastric cancer, 21 individual miRNAs and six miRNA clusters are consistently upregulated, 
whereas miR-29c, miR-30a-5p, miR-148a, miR-375 and miR-638 are typically downregulated (12-16). The biological functions of miRNAs in gastric cancer are involved in tumor formation and progression, affecting cell cycle progression, apoptosis, invasion and metastasis $(12,13,17-23)$. However, the application of these miRNAs as diagnostic or prognostic biomarkers poses a challenge.

Numerous studies have demonstrated that miRNAs are stable and detectable in human plasma, thus, indicating their potential as biomarkers for the early diagnosis of gastric cancer. Cai et al (24) measured the plasma expression levels of 15 selected miRNAs, including miR-106b, miR-20a and miR-221, and identified a statistically significant elevation in expression levels in gastric cancer patients. Furthermore, Zhang et al (25) identified that the expression levels of miR-421 in gastric cancer were significantly different compared with in benign gastric diseases, markedly improving upon the detection of early gastric cancer by using serum CEA alone. Yu et al (26) additionally determined miR-129 to be a potential biomarker for the screening of gastric cancer.

DNA hypomethylation. The epigenetic phenomenon of cancer, particularly gastric cancer, is mainly dependent on alterations in DNA methylation pattern. Global DNA hypomethylation is an early molecular event in histone protein-associated gastric carcinogenesis (27). Oishi et al (28) confirmed that the silencing of Sox 17 frequently occurs in early gastric cancer, therefore, hypermethylation of the Sox 17 gene may be applied as a useful molecular diagnostic marker in early gastric cancer (Table I).

\section{Prognostic biomarkers}

\section{Classical prognostic factors}

Microsatellite instability (MSI). MSI, resulting from errors in DNA replication, is characteristic of hereditary types of gastric cancer (29). MSI can be divided into high-level, low-level or microsatellite-stable, according to the mutation frequency. A high frequency of MSI is the result of epigenetic inactivation of the mismatch repair gene human mutL homolog 1 , whereas mutations in the transforming growth factor- $\beta$ (TGF- $\beta$ ) receptor II (RII), insulin-like growth factor-IIR and B-cell lymphoma-2 (Bcl-2)-associated X protein genes in sporadic gastric cancer are associated with a decreased propensity for invasion and nodal metastasis (30-33). Numerous studies examining the association between MSI and the prognosis of gastric cancer have been performed, the majority of which have demonstrated that MSI is associated with less aggressive behavior and more favorable survival; however, additional studies have indicated no prognostic value for MSI (34-36).

Growth factor signaling pathways and cytokines. i) Growth factors. Gastric cancer cells express a variety of growth factors and their corresponding receptors, including epidermal growth factor/receptor (EGF/R) (37), TGF- $\beta / \mathrm{R}$ and vascular endothelial cell growth factor/receptor (VEGF/R), fibroblast growth factor/receptor (bFGF/R), and platelet-derived endothelial cell growth factor/receptor (PD-ECGF/R), which are involved in tumor cell growth, angiogenesis, invasion and proliferation, respectively $(37,38)$.

EGFR and its homolog c-erbB-2 (HER2) are membrane receptors that are overexpressed in a variety of types of solid human cancer and are associated with a poor prognosis. García et al (39) identified a wide range of membranous EGFR and cytosolic HER2 expression levels in gastric cancer, which appears to be associated with the biological heterogeneity of these tumors. In addition, high tumor EGFR and HER2 expression levels were associated with an unfavorable outcome in patients with resectable gastric cancer.

However, numerous studies have indicated that HER2 is not a prognostic factor in gastric cancer. For example, Terashima et al (40) identified that overall and relapse-free survival rates were significantly lower in EGFR-positive patients compared with EGFR-negative patients, but similar in HER2-positive and -negative patients. Furthermore, Zhou et al (41) determined that the overexpression/amplification of HER-2/neu was not an independent predictor of survival in curatively resected gastric cancer patients.

Additional growth factors/receptors were determined to be associated with the prognosis of gastric cancer. For example, Kim et al (42) evaluated the expression of various growth factors/receptors in gastric adenocarcinoma, including EGFR, VEGF, VEGF-D, VEGFR-2, VEGFR-3, TGF- $\alpha$, TGF- $\beta 1$ and TGF- $\beta$ RII, using a log rank test to identify that VEGF-D and VEGFR-2 expression was associated with patient survival, and Cox regression analysis to determine that advanced stage gastric cancer and positive expression of VEGF-D were poor prognostic factors. Thus, in gastric adenocarcinoma, VEGF-D expression levels may be of prognostic value, whereas the expression levels of the EGFR and TGF family may have only a minor effect. Furthermore, high expression of the FGFR4 protein may be associated with a poor prognosis in advanced gastric cancer patients via the acceleration of disease progression (43).

In gastric cancer, the mechanism of liver metastasis may be associated with the high frequency of c-Met overexpression in the carcinoma cells; therefore, the analysis of c-Met expression levels may be a useful indicator of liver metastasis in gastric cancer patients (44). Graziano et al (45) identified that $\sim 10 \%$ of Caucasian gastric cancer patients harbored a MET gene copy number of five or above, and that this was significantly associated with an unfavorable prognosis. This data is of particular relevance to the current clinical development of anti-MET therapeutic compounds. Additionally, upregulation of the PIM-1 oncogene may be a prognostic tumor marker for gastric cancer, as PIM-1 overexpression in gastric glands has been shown to correlate with the formation of lymph node metastases and survival (46).

ii) Cytokines. Gastric cancer tumors produce various types of cytokine, including interleukin (IL)1, IL6, IL10, IL11, tumor necrosis factor (TNF), C-X-C motif chemokine $(\mathrm{CXC}) 12$, chemokine (C-C motif) ligand 1 and $\mathrm{CXC}$ receptor 2 (47-53). No mutations have been detected in key positions of glycoprotein 130 (gp130) in human gastric adenocarcinoma samples; however, gp130 activation may occur due to increased expression levels of the IL-6 and IL-11 cytokines, which have potential as valuable biomarkers (54). IL-6 induces AGS gastric cancer cell invasion via activation of the cellular-Rous sarcoma/ras homolog family member A (RhoA)/Rho-associated, coiled-coil-containing protein kinase signaling pathway, and RhoA expression may be a potential prognostic factor in gastric adenocarcinoma patients (55). 
Table I. Major molecular markers associated with the early diagnosis of gastric cancer.

\begin{tabular}{|c|c|c|}
\hline Molecule & Alteration & References \\
\hline \multicolumn{3}{|l|}{ Classical biomarker } \\
\hline CEA & Increase & 10 \\
\hline CA19-9 & Increase & 11 \\
\hline \multicolumn{3}{|l|}{ miRNA } \\
\hline miR-106b, miR-20a, miR-221, miR-421, miR-129 & Upregulation & $12-16,24-26$ \\
\hline \multicolumn{3}{|l|}{ DNA hypomethylation } \\
\hline Sox 17 & Downregulation & 28 \\
\hline
\end{tabular}

CEA, carcinoembryonic antigen; CA19-9, cancer antigen19-9; miR/miRNA, microRNA.

Table II. Major molecular markers associated with the prognosis of gastric cancer.

\begin{tabular}{|c|c|c|}
\hline Marker & Alteration & References \\
\hline MSI & High level & $30-36$ \\
\hline \multicolumn{3}{|l|}{ Growth factors } \\
\hline EGFR, HER-2, VEGF, TGF, c-MET & Overexpression & $39-44$ \\
\hline \multicolumn{3}{|l|}{ Cytokines } \\
\hline IL-6, IL-11 & Upregulation & 54,55 \\
\hline \multicolumn{3}{|l|}{ Cell cycle regulators } \\
\hline Cyclin E & Overexpression & $59-61$ \\
\hline \multicolumn{3}{|l|}{ Apoptosis-associated factors } \\
\hline Bcl-2, Fas, survivin & Overexpression & $67-70$ \\
\hline \multicolumn{3}{|l|}{ miRNAs } \\
\hline Let-7g, miR-433 & Downregulation & 15 \\
\hline miR-214, miR-21 & Upregulation & 15,77 \\
\hline \multicolumn{3}{|l|}{ Epigenetic alterations } \\
\hline Runx3, E-cadherin, WNT5A & Hypermethylation & $83-85$ \\
\hline \multicolumn{3}{|l|}{ Genetic polymorphisms } \\
\hline p53, IL-1, IL-10 & SNP & $104-106$ \\
\hline
\end{tabular}

MSI, microsatellite instability; EGFR, epidermal growth factor receptor; HER-2, human epidermal growth factor receptor-2; VEGF, vascular endothelial growth factor; TGF, transforming growth factor $\beta$; IL, interleukin; Bcl-2, B-cell lymphoma-2; miR/miRNA, microRNA; Runx3, runt-related transcription factor 3; WNT5A, wingless-type MMTV integration site family, member 5A; SNP, single-nucleotide polymorphism.

Additionally, IL-12-positive cell density may be a significant independent prognostic factor in the analysis of advanced gastric cancer surgical specimens (50); and chemokine (C-C motif) receptor 4 and its ligands appear to be associated with increased tumor recurrence and impaired overall survival in gastric cancer patients (56).

Cell cycle factors and apoptosis. i) Cell cycle regulators. Abnormalities in cell-cycle regulators are associated with various aspects of gastric cancer, including cancer cell proliferation. Furthermore, specific cell cycle factors are associated with the prognosis of gastric cancer (57), for example, cyclins condition the progression of the cell cycle by activating appropriate serine-threonine kinases. Therefore, changes in cyclin expression levels lead to pathologies of cell division, including neoplastic proliferation. The activity of cyclins D1 and E when complexed with appropriate cyclin-dependent kinases may be inhibited by protein p21 (WAF1/CIP1), which functions as an inhibitor of the cell growth cycle (58). Of the two types of cyclin evaluated, only cyclin $\mathrm{E}$ is considered to be a significant regulatory factor and useful prognostic parameter in gastric cancer; furthermore, it has been demonstrated that reduced p27 expression is a negative prognostic factor for patients with cyclin E-positive tumors (59-61). Similarly, alterations in the p53 gene are associated with less favorable prognoses in advanced gastric cancer; this may be by the potential provision of vertical growth into the gastric wall. Multivariate analysis demonstrated that the overexpression of p53 was an independent prognostic factor for advanced gastric cancer patients (62), however, the expression 
of p53 alone exhibited no prognostic value for early gastric cancer patients (63). Additionally, the poor prognosis for patients with low retinoblastoma protein ( $\mathrm{pRb}$ ) expression levels and decreased $\mathrm{pRb}$ expression in lymph node metastases indicate that $\mathrm{Rb}$ and its associated genes may affect cancer progression (64).

ii) Apoptosis-associated factors. Apoptosis is an established type of programmed cell death. Abnormalities in apoptosis promote gastric carcinogenesis (65) and numerous apoptosis-associated factors have been determined to be prognostic indicators of gastric cancer (66-73). For example, the $\mathrm{Bcl}-2$ proto-oncogene is important in determining the susceptibility of tumor cells to apoptosis; with Bcl-2 expression and a high apoptotic fraction determined to be important prognostic factors of survival in advanced gastric cancer patients $(67,68)$. Fas (apoptosis-1/cluster of differentiation 95), a member of the TNF/nerve growth factor receptor superfamily, mediates apoptosis as a response to agonistic antibodies or Fas ligand (FasL) binding. Recently, it was reported that tumor cells are able to express FasL, inducing apoptosis in tumor-infiltrating lymphocytes and thus allowing them to escape host immune surveillance. In gastric carcinoma, tumor progression via the lymphatics is often observed, and lymph node metastasis is a critical factor affecting the recurrence and prognosis of cancer; previous studies have demonstrated that upregulation of FasL may correlate with this progression of gastric carcinoma $(69,70)$. In addition, survivin is a recently characterized gene and a member of the inhibition of apoptosis family that inhibits apoptosis via pathways that do not involve the Bcl-2 family (74-76). Specific studies have indicated that survivin is present in the majority of gastric cancer cells, however, the nuclear localization of survivin appears to be physiologically important in hindering tumor progression (77), therefore, survivin may be an important predictive and prognostic parameter of poor outcome in gastric carcinoma (78).

\section{Novel prognostic factors}

miRNA. miRNAs may be used as prognostic factors, as they are expressed in stable and robust levels in tissues and the circulation (74-76). In particular, miRNAs have been associated with survival times and disease stage in gastric cancer patients, as well as with tumor recurrence and metastasis to the lymph nodes.

Ueda et al (15) identified that low let-7 g and miR-433 expression levels, and high miR-214 expression levels were associated with unfavorable overall survival outcomes, independent of clinical covariates, including invasion depth, lymph-node, tumor stage and metastasis. An additional study confirmed that high miR-20a, miR-25, miR-93, miR-103, miR-106a, miR-106b, miR-130, miR-155, miR-221 and miR-222 expression levels in advanced gastric cancer tissues appeared to be risk factors highly associated with the penetration of the tumor through the serosa, distant metastasis, lymph node metastasis and poor long-term survival in those patients undergoing radical resection procedures and adjuvant systemic chemotherapy (79).

Circulating miRNA, which is stable and easy to obtain, was previously considered to be a promising biomarker for predicting survival in gastric cancer. For example, Komatsu et al (74) identified that a high miR-21 concentration in plasma was an independent prognostic factor in gastric cancer, and another study detected increased miR-200c levels in the blood of gastric cancer patients, indicating that miR-200c may be also a potential predictor of gastric cancer progression and survival (80). However, these studies of miRNAs as prognostic factors involved small sample sets; thus, validation in larger, independent cohorts is required prior to the application of miRNA assays in a clinical setting.

Epigenetic alterations. Genetic and epigenetic mechanisms are involved in molecular alterations and pathway dysregulation. In contrast to genetic alterations, epigenetic changes, such as DNA methylation and histone modifications, affect the phenotypic outcomes of a genome without changing the underlying DNA sequences (81).

The hypermethylation of $\mathrm{CpG}$ islands is associated with the silencing of tumor suppressor genes and is involved in tumorigenesis. The hypermethylation of various genes, such as cadherin 1 (CDH1) (82), coiled-coil domain containing protein 67 (83), methylated in tumors 31 (84), p16 (85), runt-related transcription factor 3 (85), E-cadherin (86), hMHL1 (87) and wingless-type MMTV integration site family, member 5A (88), has been identified to be associated with the prognosis of gastric cancer. However, not all methylation acts as a prognostic marker. Raf kinase inhibitory protein (RKIP) has been identified to be a member of a novel molecular class that has been indicated to be involved in cancer progression and the suppression of metastatic tumor spread; therefore, hypermethylation and loss of RKIP expression may be used as a marker to predict the clinical outcome of advanced gastric cancer (89). Similarly, methylated $\mathrm{CDH} 1$ predicts a poor prognosis in gastric cancer patients (82); however, S100A6 is important in the progression and prognosis of gastric cancer, and is upregulated by epigenetic regulation (90). Methylation of Bcl-2/adenovirus E1B 19-kDa-interacting protein 3 and death-associated protein kinase can predict a reduced response to chemotherapy and a poor prognosis in gastric cancer (91). Furthermore, paired box 5 (PAX5) is a novel functional tumor suppressor in gastric carcinogenesis, and detection of methylated PAX5 can be utilized as an independent prognostic factor in gastric cancer (92).

Genetic polymorphisms. Genetic polymorphisms appear to be an important cause of gastric carcinogenesis, with genetic susceptibility associated with gastric cancer risk. Various studies have demonstrated that the presence of numerous single nucleotide polymorphisms (SNPs) is associated with increased gastric cancer susceptibility; for example, excision repair cross-complementation group 1, CDH1, IL-10, IL-1, IL-6, VEGF and FAS gene SNPs have been found to be associated with the risk of gastric cancer (Table II) (93-101).

Additionally, specific SNPs appear to predict the outcome of chemotherapy or adjuvant chemotherapy treatment strategies. For example, polymorphisms of rs1801159 in dihydropyrimidine dehydrogenase (DPD), a key enzyme involved in the catabolism of 5-fluorouracil (5-FU), may be utilized as valuable FU-based chemotherapy response predictors for patients with gastric cancer in the Chinese population (102). Furthermore, the G/G genotype of the VEGF-634 G/C polymorphism is associated with higher serum expression levels of VEGF and a poor clinical outcome in patients with advanced gastric cancer who are treated with oxaliplatin, 5-FU and 
leucovorin (103). In addition, polymorphisms of glutathione $S$-transferase $\mathrm{P} 1$, xeroderma pigmentosum group $\mathrm{D}$ and $\mathrm{X}$-ray repair cross complementing group 1 have been shown to predict the clinical outcome of gastric cancer patients to oxaliplatin/5-FU-based chemotherapy $(104,105)$. The tumor protein p53 (TP53) codon 72 SNP was determined to be predictive of the response to chemotherapy, and correlated with the time to progression in advanced gastric cancer patients treated with paclitaxel and cisplatin chemotherapy (106).

Furthermore, various SNPs were associated with the prognosis of gastric cancer. Shirai et al (107) determined that the p53 SNP Arg72Pro was associated with a poor prognosis in gastric cancer (107), while Kim et al (106) identified that the TP53 codon 72 SNP was predictive of the chemotherapy response and that it was associated with the time to progression in advanced gastric cancer patients treated with paclitaxel and cisplatin chemotherapy. A different study determined that the TP53 codon 72 polymorphism was associated with gastric cancer patient survival in those treated with 5-FU-based post-operative chemotherapy (108); thus, the TP53 codon 72 polymorphism may be a potential prognostic factor in gastric cancer.

Numerous studies have been conducted to investigate the effect of cytokines and other pro-inflammatory mediator gene polymorphisms on the prognosis of gastric cancer, however, controversy remains. For example, García-González et al (109) determined that pro- and anti-inflammatory cytokine gene polymorphisms, such as IL1B, TNFA, LTA, IL6, IL12p40, IL4, IL1RN, IL10 and TGFB1, may not be relevant in determining the prognosis of gastric adenocarcinoma patients. Similarly, Liu et al (110) identified that IL-10 gene promoter polymorphisms may not be associated with the prognosis of advanced gastric cancer. By contrast, Tahara et al (111) proposed that the IL-1 $\beta-31 C C$ and IL-1 $\beta-511$ TT genotypes, and the TNF- $\alpha-857 \mathrm{~T}$ carrier may exhibit a protective effect against gastric cancer progression.

\section{Conclusion}

Gastric cancer is a noteworthy disease due to its heterogeneous properties. Although the molecular basis of gastric cancer has been thoroughly investigated, resulting in significant progression within the field, ubiquitous biomarkers are rare. Therefore, the identification of novel and specific markers to diagnose and predict gastric cancer survival is important.

With the development of modern technologies, such as genome and exome sequencing, and miRNA microarrays, various novel biomarkers with diagnostic and prognostic value have been identified. Although complicated, clinical trials for the screening of novel biomarkers prior to clinical application have been necessary, and thus far have resulted in promising outcomes.

\section{Acknowledgements}

The present study was supported by a grant from the National Natural Science Foundation of China (no. 81071667).

\section{References}

1. Jemal A, Bray F, Center MM, et al: Global cancer statistics. CA Cancer J Clin 61: 69-90, 2011.
2. Hu B, El Hajj N, Sittler S, et al: Gastric cancer: Classification, histology and application of molecular pathology. J Gastrointest Oncol 3: 251-261, 2012.

3. Nitti D, Mocellin S, Marchet A, Pilati P and Lise M: Recent advances in conventional and molecular prognostic factors for gastric carcinoma. Surg Oncol Clin N Am 17: 467-483, 2008.

4. Resende C, Ristimäki A and Machado JC: Genetic and epigenetic alteration in gastric carcinogenesis. Helicobacter 15: 34-39, 2010.

5. Pectasides D, Mylonakis A, Kostopoulou M, et al: CEA, CA 19-9, and CA-50 in monitoring gastric carcinoma. Am J Clin Oncol 20: 348-353, 1997.

6. Wu WK, Lee CW, Cho CH, et al: MicroRNA dysregulation in gastric cancer: a new player enters the game. Oncogene 29: 5761-5771, 2010.

7. Ye T, Chen Y and Fang J: DNA methylation biomarkers in serum for gastric cancer screening, Mini Rev Med Chem 10: 1034-1038, 2010.

8. Lu J, Huang CM, Zheng CH, et al: Prognostic value of tumor size in patients with remnant gastric cancer: Is the seventh UICC stage sufficient for predicting prognosis? PloS One 9: e115776, 2014.

9. Gold P and Freedman SO: Specific carcinoembryonic antigens of the human digestive system. J Exp Med 122: 467-481, 1965.

10. Tatsuta M, Itoh T, Okuda S, et al: Carcinoembryonic antigen in gastric juice as an aid in diagnosis of early gastric cancer. Cancer 46: 2686-2692, 1980.

11. Szymendera JJ: Clinical usefulness of three monoclonal antibody-defined tumor markers: CA 19-9, CA 50, and CA 125. Tumour Biol 7: 333-342, 1986.

12. Tsukamoto Y, Nakada C, Noguchi T, et al: MicroRNA-375 is downregulated in gastric carcinomas and regulates cell survival by targeting PDK1 and 14-3-3zeta. Cancer Res 70: 2339-2349, 2010.

13. Oh HK, Tan AL, Das K, et al: Genomic loss of miR-486 regulates tumor progression and the OLFM4 antiapoptotic factor in gastric cancer. Clin Cancer Res 17: 2657-2667, 2011.

14. Katada T, Ishiguro H, Kuwabara Y, et al: microRNA expression profile in undifferentiated gastric cancer. Int J Oncol 34: 537-542, 2009.

15. Ueda T, Volinia S, Okumura H, et al: Relation between microRNA expression and progression and prognosis of gastric cancer: a microRNA expression analysis. Lancet Oncol 11: 136-146, 2010.

16. Song JH and Meltzer SJ: MicroRNAs in pathogenesis, diagnosis, and treatment of gastroesophageal cancers. Gastroenterology 143: 35-47.e2, 2012

17. He XP, Shao Y, Li XL, et al: Downregulation of miR-101 in gastric cancer correlates with cyclooxygenase-2 overexpression and tumor growth. FEBS J 279: 4201-4212, 2012.

18. Wang M, Li C, Nie H, et al: Down-regulated miR-625 suppresses invasion and metastasis of gastric cancer by targeting ILK. FEBS Lett 586: 2382-2388, 2012.

19. Yamanaka S, Olaru AV, An F, et al: MicroRNA-21 inhibits Serpini1, a gene with novel tumour suppressive effects in gastric cancer. Dig Liver Dis 44: 589-596, 2012.

20. Cao W, Fan R, Wang L, et al: Expression and regulatory function of miRNA-34a in targeting survivin in gastric cancer cells. Tumour Biol 34: 963-971, 2013.

21. Crone SG, Jacobsen A, Federspiel B, et al: microRNA-146a inhibits $G$ protein-coupled receptor-mediated activation of NF- $\kappa \mathrm{B}$ by targeting CARD10 and COPS8 in gastric cancer. Mol Cancer 11: 71,2012.

22. Sacconi A, Biagioni F, Canu V, et al: miR-204 targets $\mathrm{Bcl}-2$ expression and enhances responsiveness of gastric cancer. Cell Death Dis 3: e423, 2012.

23. Xu Y, Zhao F, Wang Z, et al: MicroRNA-335 acts as a metastasis suppressor in gastric cancer by targeting Bcl-w and specificity protein 1. Oncogene 31: 1398-1407, 2012.

24. Cai H, Yuan Y, Hao YF, et al: Plasma microRNAs serve as novel potential biomarkers for early detection of gastric cancer. Med Oncol 30: 452, 2013

25. Zhang X, Cui L, Ye G, et al: Gastric juice microRNA-421 is a new biomarker for screening gastric cancer. Tumour Biol 33: 2349-2355, 2012

26. Yu X, Luo L, Wu Y, et al: Gastric juice miR-129 as a potential biomarker for screening gastric cancer. Med Oncol 30: 365, 2013.

27. Compare D, Rocco A, Liguori E, et al: Global DNA hypomethylation is an early event in Helicobacter pylori-related gastric carcinogenesis. J Clin Pathol 64: 677-682, 2011.

28. Oishi Y, Watanabe Y, Yoshida Y, et al: Hypermethylation of Sox17 gene is useful as a molecular diagnostic application in early gastric cancer. Tumour Biol 33: 383-393, 2012. 
29. Hudler P: Genetic aspects of gastric cancer instability. ScientificWorldJournal 2012: 761909, 2012

30. Ling ZQ, Tanaka A, Li P, et al: Microsatellite instability with promoter methylation and silencing of hMLH1 can regionally occur during progression of gastric carcinoma. Cancer Lett 297: 244-251, 2010

31. Ohue M, Tomita N,Monden T, et al: Mutations of the transforming growth factor beta type II receptor gene and microsatellite instability in gastric cancer. Int J Cancer 68: 203-206, 1996.

32. Iacopetta BJ, Soong R, House AK and Hamelin R: Gastric carcinomas with microsatellite instability: clinical features and mutations to the TGF-beta type II receptor, IGFII receptor, and BAX genes. J Pathol 187: 428-432, 1999.

33. Fleisher AS, Esteller M, Wang S, et al: Hypermethylation of the hMLH1 gene promoter in human gastric cancers with microsatellite instability. Cancer Res 59: 1090-1095, 1999.

34. Choi SW, Choi JR, Chung YJ, et al: Prognostic implications of microsatellite genotypes in gastric carcinoma. Int J Cancer 89: 378-383, 2000

35. Wirtz HC, Müller W, Noguchi T, et al: Prognostic value and clinicopathological profile of microsatellite instability in gastric cancer. Clin Cancer Res 4: 1749-1754, 1998.

36. dos Santos NR, Seruca R, Constância M, et al: Microsatellite instability at multiple loci in gastric carcinoma: clinicopathologic implications and prognosis. Gastroenterology 110: 38-44, 1996.

37. Yasui W, Sumiyoshi H, Hata J, et al: Expression of epidermal growth factor receptor in human gastric and colonic carcinomas. Cancer Res 48: 137-141, 1988.

38. Sakai K, Mori S, Kawamoto T, et al: Expression of epidermal growth factor receptors on normal human gastric epithelia and gastric carcinomas. J Natl Cancer Inst 77: 1047-1052, 1986.

39. García I, Vizoso F, Martín A, et al: Clinical significance of the epidermal growth factor receptor and HER2 receptor in resectable gastric cancer. Ann Surg Oncol 10: 234-241, 2003

40. Terashima M, Kitada K, Ochiai A, et al; ACTS-GC Group: Impact of expression of human epidermal growth factor receptors EGFR and ERBB2 on survival in stage II/III gastric cancer. Clin Cancer Res 18: 5992-6000, 2012.

41. Zhou F, Li N, Jiang W, et al: Prognosis significance of HER-2/neu overexpression/amplification in Chinese patients with curatively resected gastric cancer after the ToGA clinical trial. World J Surg Oncol 10: 274, 2012.

42. Kim JY, Jeon TJ, Bae BN, et al: The prognostic significance of growth factors and growth factor receptors in gastric adenocarcinoma. APMIS 121: 95-104, 2013.

43. Ye YW, Zhang X, Zhou Y, et al: The correlations between the expression of FGFR4 protein and clinicopathological parameters as well as prognosis of gastric cancer patients. J Surg Oncol 106: 872-879, 2012

44. Amemiya $\mathrm{H}$, Kono $\mathrm{K}$, Itakura J, et al: c-Met expression in gastric cancer with liver metastasis. Oncology 63: 286-296, 2002.

45. Graziano F, Galluccio N, Lorenzini P, et al: Genetic activation of the MET pathway and prognosis of patients with high-risk, radically resected gastric cancer. J Clin Oncol 29: 4789-4795, 2011.

46. Warnecke-Eberz U, Bollschweiler E, Drebber U, et al: Prognostic impact of protein overexpression of the proto-oncogene PIM-1 in gastric cancer. Anticancer Res 29: 4451-4455, 2009.

47. Ishigami S, Natsugoe $\mathrm{S}$, Okumura $\mathrm{H}$, et al: Clinical implication of CXCL12 expression in gastric cancer. Ann Surg Oncol 14: 3154-3158, 2007

48. Kim DK, Oh SY, Kwon HC, et al: Clinical significances of preoperative serum interleukin-6 and C-reactive protein level in operable gastric cancer. BMC Cancer 9: 155, 2009.

49. Liao WC, Lin JT, Wu CY, et al: Serum interleukin-6 level but not genotype predicts survival after resection in stages II and III gastric carcinoma. Clin Cancer Res 14: 428-434, 2008.

50. Nagashima N, Nakayama Y, Inoue Y, et al: Prognostic significance of the local expression of interleukin-12 in patients with advanced gastric cancer. Anticancer Res 28: 1277-1283, 2008.

51. Ashizawa T, Okada R, Suzuki Y, et al: Clinical significance of interleukin-6 (IL-6) in the spread of gastric cancer: role of IL-6 as a prognostic factor. Gastric Cancer 8: 124-131, 2005.

52. Graziano F, Ruzzo A, Santini D, et al: Prognostic role of interleukin-1beta gene and interleukin-1 receptor antagonist gene polymorphisms in patients with advanced gastric cancer. J Clin Oncol 23: 2339-2345, 2005 .

53. Macrì A, Versaci A, Loddo S, et al: Serum levels of interleukin 1beta, interleukin 8 and tumour necrosis factor alpha as markers of gastric cancer. Biomarkers 11: 184-193, 2006.
54. Necula LG, Chivu-Economescu M, Stanciulescu EL, et al: IL- 6 and IL-11 as markers for tumor aggressiveness and prognosis in gastric adenocarcinoma patients without mutations in Gp130 subunits. J Gastrointestin Liver Dis 21: 23-29, 2012.

55. Lin MT, Lin BR, Chang CC, et al: IL-6 induces AGS gastric cancer cell invasion via activation of the $\mathrm{c}-\mathrm{Src} / \mathrm{RhoA} / \mathrm{ROCK}$ signaling pathway. Int J Cancer 120: 2600-2608, 2007

56. Lee JH, Cho YS, Lee JY, et al: The chemokine receptor CCR4 is expressed and associated with a poor prognosis in patients with gastric cancer. Ann Surg 249: 933-941, 2009.

57. Al-Moundhri MS, Nirmala V, Al-Hadabi I, et al: The prognostic significance of p53, p27 kip1, p21 waf1, HER-2/neu, and Ki67 proteins expression in gastric cancer: a clinicopathological and immunohistochemical study of 121 Arab patients. J Surg Oncol 91: 243-252, 2005.

58. Tenderenda M: A study on the prognostic value of cyclins D1 and E expression levels in resectable gastric cancer and on some correlations between cyclins expression, histoclinical parameters and selected protein products of cell-cycle regulatory genes. J Exp Clin Cancer Res 24: 405-414, 2005.

59. Nitti D, Belluco C, Mammano E, et al: Low level of p27(Kip1) protein expression in gastric adenocarcinoma is associated with disease progression and poor outcome. J Surg Oncol 81: 167-175; discussion 175-166, 2002.

60. Xiangming C, Natsugoe S, Takao S, et al: The cooperative role of p27 with cyclin E in the prognosis of advanced gastric carcinoma. Cancer 89: 1214-1219, 2000.

61. Sgambato A, Migaldi M, Leocata P, et al: Loss of p27Kip1 expression is a strong independent prognostic factor of reduced survival in N0 gastric carcinomas. Cancer 89: 2247-2257, 2000.

62. Ichiyoshi Y, Oiwa H, Tomisaki S, et al: Overexpression of $\mathrm{p} 53$ is associated with growth pattern and prognosis in advanced gastric cancer. Hepatogastroenterology 44: 546-553, 1997.

63. Gonçalves AR, Carneiro AJ, Martins I, et al: Prognostic significance of p53 protein expression in early gastric cancer. Pathol Oncol Res 17: 349-355, 2011.

64. Feakins RM, Nickols CD, Bidd H and Walton SJ: Abnormal expression of $\mathrm{pRb}, \mathrm{p} 16$, and cyclin $\mathrm{D} 1$ in gastric adenocarcinoma and its lymph node metastases: relationship with pathological features and survival. Hum Pathol 34: 1276-1282, 2003.

65. Aurello P, Bellagamba R, Rossi Del Monte S, et al: Apoptosis and microvessel density in gastric cancer: correlation with tumor stage and prognosis. Am Surg 75: 1183-1188, 2009.

66. Koshida Y, Saegusa M and Okayasu I: Apoptosis, cell proliferation and expression of $\mathrm{Bcl}-2$ and $\mathrm{Bax}$ in gastric carcinomas: immunohistochemical and clinicopathological study. Br J Cancer 75: 367-373, 1997.

67. Inada T, Kikuyama S, Ichikawa A, et al: Bcl-2 expression as a prognostic factor of survival of gastric carcinoma. Anticancer Res 18: 2003-2010, 1998.

68. Aizawa K, Ueki K, Suzuki S, et al: Apoptosis and Bbcl-2 expression in gastric carcinomas: correlation with clinicopathological variables, p53 expression, cell proliferation and prognosis. Int J Oncol 14: 85-91, 1999.

69. Osaki M, Kase S, Kodani I, et al: Expression of Fas and Fas ligand in human gastric adenomas and intestinal-type carcinomas: correlation with proliferation and apoptosis. Gastric Cancer 4: 198-205, 2001

70. Nagashima H, Mori M, Sadanaga N, et al: Expression of Fas ligand in gastric carcinoma relates to lymph node metastasis. Int J Oncol 18: 1157-1162, 2001.

71. Liu X, Cai H, Huang H, et al: The prognostic significance of apoptosis-related biological markers in Chinese gastric cancer patients. PLoS One 6: e29670,2011.

72. Lee HK, Lee HS, Yang HK, et al: Prognostic significance of Bcl-2 and $\mathrm{p} 53$ expression in gastric cancer. Int J Colorectal Dis 18: $518-525,2003$.

73. Kim MA, Lee HE, Lee HS, et al: Expression of apoptosis-related proteins and its clinical implication in surgically resected gastric carcinoma. Virchows Arch 459: 503-510, 2011.

74. Komatsu S, Ichikawa D, Tsujiura M, et al: Prognostic impact of circulating miR-21 in the plasma of patients with gastric carcinoma. Anticancer Res 33: 271-276, 2013

75. Cui L, Zhang X, Ye G, et al: Gastric juice MicroRNAs as potential biomarkers for the screening of gastric cancer. Cancer 119: 1618-1626, 2013.

76. Liu JL, Gao W, Kang QM, et al: Prognostic value of survivin in patients with gastric cancer: a systematic review with metaanalysis. PLoS One 8: e71930, 2013. 
77. Okada E, Murai Y, Matsui K, et al: Survivin expression in tumor cell nuclei is predictive of a favorable prognosis in gastric cancer patients. Cancer Lett 163: 109-116, 2001.

78. Song KY, Jung CK, Park WS and Park CH: Expression of the antiapoptosis gene Survivin predicts poor prognosis of stage III gastric adenocarcinoma. Jpn J Clin Oncol 39: 290-296, 2009.

79. Kim BH, Hong SW, Kim A, et al: Prognostic implications for high expression of oncogenic microRNAs in advanced gastric carcinoma. J Surg Oncol 107: 505-510, 2013.

80. Valladares-Ayerbes M, Reboredo M, Medina-Villaamil V, et al: Circulating miR-200c as a diagnostic and prognostic biomarker for gastric cancer. J Transl Med 10: 186, 2012.

81. Cheng AS, Li MS, Kang W, et al: Helicobacter pylori causes epigenetic dysregulation of FOXD3 to promote gastric carcinogenesis. Gastroenterology 144: 122-133.e9, 2013.

82. Yu QM, Wang XB, Luo J, et al: CDH1 methylation in preoperative peritoneal washes is an independent prognostic factor for gastric cancer. J Surg Oncol 106: 765-771, 2012.

83.Park SJ, Jang HR, Kim M, et al: Epigenetic alteration of CCDC67 and its tumor suppressor function in gastric cancer. Carcinogenesis 33: 1494-1501, 2012.

84.Negrini M, Miotto E, Sabbioni S, et al: MINT31 methylation in gastric noninvasive neoplasia: potential role in the secondary prevention of gastric cancer. Eur J Cancer Prev 21 442-448, 2012

85.Hu SL, Kong XY, Cheng ZD, et al: Promoter methylation of p16, Runx3, DAPK and CHFR genes is frequent in gastric carcinoma. Tumori 96: 726-733, 2010.

86. Huang FY, Chan AO, Rashid A, et al: Helicobacter pylori induces promoter methylation of E-cadherin via interleukin- $1 \beta$ activation of nitric oxide production in gastric cancer cells. Cancer 118: 4969-4980, 2012.

87. Wani M, Afroze D, Makhdoomi M, et al: Promoter methylation status of DNA repair gene (hMLH1) in gastric carcinoma patients of the Kashmir valley. Asian Pac J Cancer Prev 13: 4177-4181, 2012

88. Hibi K, Sakata M, Yokomizi K, et al: Methylation of the WNT5A gene is frequently detected in early gastric carcinoma. Hepatogastroenterology 59: 2661-2663, 2012.

89. Guo W, Dong Z, Guo Y, et al: Aberrant methylation and loss expression of RKIP is associated with tumor progression and poor prognosis in gastric cardia adenocarcinoma. Clin Exp Metastasis 30: 265-275, 2013.

90. Wang XH, Zhang LH, Zhong XY, et al: S100A6 overexpression is associated with poor prognosis and is epigenetically up-regulated in gastric cancer. Am J Pathol 177: 586-597, 2010.

91. Sugita H, Iida S, Inokuchi M, et al: Methylation of BNIP3 and DAPK indicates lower response to chemotherapy and poor prognosis in gastric cancer. Oncol Rep 25: 513-518, 2011.

92. Li X, Cheung KF, Ma X, et al: Epigenetic inactivation of paired box gene 5, a novel tumor suppressor gene, through direct upregulation of p53 is associated with prognosis in gastric cancer patients. Oncogene 31: 3419-3430, 2012.

93. Zhan Z, Wu J, Zhang JF, et al: CDH1 gene polymorphisms, plasma $\mathrm{CDH} 1$ levels and risk of gastric cancer in a Chinese population. Mol Biol Rep 39: 8107-8113, 2012.

94. Yuan LJ, Jin TB, Yin JK, et al: Polymorphisms of tumor-related genes IL-10, PSCA, MTRR and NOC3 L are associated with the risk of gastric cancer in the Chinese Han population. Cancer Epidemiol 36: e366-e372, 2012.

95. Xue H, Lu Y, Lin B, et al: The effect of XPD/ERCC2 polymorphisms on gastric cancer risk among different ethnicities: a systematic review and meta-analysis. PLoS One 7: e43431, 2012.
96. Tian J, Pan F, Li J, et al: Association between the FAS/FASL polymorphisms and gastric cancer risk: a meta-analysis. Asian Pac J Cancer Prev 13: 945-951, 2012

97. Ozen A, Kocak Z, Sipahi T, et al: The prognostic significance of p21 and Her-2 gene expression and mutation/polymorphism in patients with gastric adenocarcinoma. Med Oncol 30: 357, 2013.

98. Lu R, Gao X, Chen Y, et al: Association of an NFKB1 intron SNP (rs4648068) with gastric cancer patients in the Han Chinese population. BMC Gastroenterol 12: 87, 2012.

99. He J, Xu Y, Qiu LX, et al: Polymorphisms in ERCC1 and XPF genes and risk of gastric cancer in an eastern Chinese population. PLoS One 7: e49308, 2012.

100. Han L, Lee SW, Yoon JH, et al: Association of SOD1 and SOD2 single nucleotide polymorphisms with susceptibility to gastric cancer in a Korean population. APMIS 121: 246-256, 2013.

101.Han F, Wang X, Wang X, Luo Y and Li W: Meta-analysis of the association of CYP1A1 polymorphisms with gastric cancer susceptibility and interaction with tobacco smoking. Mol Biol Rep 39: 8335-8344, 2012.

102.Zhang XP, Bai ZB, Chen BA, et al: Polymorphisms of dihydropyrimidine dehydrogenase gene and clinical outcomes of gastric cancer patients treated with fluorouracil-based adjuvant chemotherapy in Chinese population. Chin Med J (Engl) 125: 741-746, 2012

103. Oh SY, Kwon HC, Kim SH, et al: The relationship of vascular endothelial growth factor gene polymorphisms and clinical outcome in advanced gastric cancer patients treated with FOLFOX: VEGF polymorphism in gastric cancer. BMC Cancer 13: 43, 2013.

104.Liu B, Wei J, Zou Z, et al: Polymorphism of XRCC1 predicts overall survival of gastric cancer patients receiving oxaliplatin-based chemotherapy in Chinese population. Eur J Hum Genet 15: 1049-1053, 2007.

105.Li QF, Yao RY,Liu KW, et al: Genetic polymorphism of GSTP1: prediction of clinical outcome to oxaliplatin/5-FU-based chemotherapy in advanced gastric cancer. J Korean Med Sci 25: 846-852, 2010.

106. Kim JG, Sohn SK, Chae YS, et al: TP53 codon 72 polymorphism associated with prognosis in patients with advanced gastric cancer treated with paclitaxel and cisplatin. Cancer Chemother Pharmacol 64: 355-360, 2009

107. Shirai O,Ohmiya N, Taguchi A, et al:P53,p21, and p73 gene polymorphisms in gastric carcinoma. Hepatogastroenterology 57 . 1595-1601, 2010.

108. Wang S, Chen L, Zhao Q, et al: Effect of TP53 codon 72 and MDM2 SNP309 polymorphisms on survival of gastric cancer among patients who receiving 5-fluorouracil-based postoperative adjuvant chemotherapy. Cancer Chemother Pharmacol 71: 1073-1082, 2013.

109. García-González MA, Nicolás-Pérez D, Lanas A, et al: Prognostic role of host cyclooxygenase and cytokine genotypes in a Caucasian cohort of patients with gastric adenocarcinoma. PLoS One 7: e46179, 2012.

110. Liu J, Song B, Wang JL, et al: Polymorphisms of interleukin-10 promoter are not associated with prognosis of advanced gastric cancer. World J Gastroenterol 17: 1362-1367, 2011.

111. Tahara T, Shibata T, Nakamura M, et al: Effect of IL-1 $\beta$ and TNF- $\alpha$ polymorphisms on the prognosis and survival of gastric cancer patients. Clin Exp Med 11: 211-217, 2011. 